\title{
The Multidrug Transporters Belonging to Major Facilitator Superfamily (MFS) in Mycobacterium tuberculosis
}

\author{
Edda De Rossi, ${ }^{1}$ Patrizio Arrigo, ${ }^{2}$ Marco Bellinzoni, ${ }^{1}$ Pedro E. A. Silva, ${ }^{3,5}$ Carlos Martín, ${ }^{3}$ \\ José A. Aínsa, ${ }^{3}$ Paola Guglierame, ${ }^{4}$ and Giovanna Riccardi ${ }^{4}$ \\ ${ }^{1}$ Dipartimento di Genetica e Microbiologia, Università degli Studi di Pavia, Pavia, Italy \\ ${ }^{2}$ Istituto Circuiti Elettronici, CNR, Genova, Italy \\ ${ }^{3}$ Departamento de Microbiología Medicina Preventiva y Salud Pública, Universidad de Zaragoza, \\ Zaragoza, Spain \\ ${ }^{4}$ Dipartimento di Biologia Sperimentale Ambientale ed Applicata, Università degli Studi di Genova, \\ Genova, Italy \\ ${ }^{5}$ Present address: Fundaçaõ Universidade Federal do Rio Grande, Brasil \\ Accepted September 24, 2002
}

\begin{abstract}
Background: Both intrinsic and acquired multidrug resistance play an important role in the insurgence of tuberculosis. Detailed knowledge of the molecular basis of drug recognition and transport by multidrug transport systems is required for the development of new antibiotics that are not extruded or of inhibitors that block the multidrug transporter and allow traditional antibiotics to be effective. Materials and Methods: We have undertaken the inventory of the drug transporters subfamily, included in the major facilitator superfamily (MFS), encoded by the com-
\end{abstract}

plete genome of Mycobacterium tuberculosis (MTB). These proteins were identified on the basis of their characteristic stretches of amino acids and transmembrane segments (TMS) number.

Conclusions: Genome analysis and searches of homology between the identified transporters and proteins characterized in other organisms revealed 16 open reading frames encoding putative drug efflux pumps belonging to MFS. In the case of two of them, we also have demonstrated that they function as drug efflux proteins.

\section{Introduction}

One of the major problems in combating tuberculosis disease is that Mycobacterium tuberculosis is resistant to most therapeutic agents because it has an unusual bacterial cell wall with intrinsically low permeability (1).

Although acquired high-level drug resistance in mycobacteria in general is due to mutational alterations of the drug target, it has become clear that low-level drug resistance to a variety of different anti-infective agents is frequently found in clinically drug-resistant isolates with no alteration of the target. Nothing is presently known about the molecular mechanisms mediating this class of resistance, although it is likely that changes in drug transport are involved (2).

Multidrug efflux systems endow on bacterial cells the ability to limit the access of antimicrobial agents to their targets. Drug efflux appears to be one of the most widespread antibiotic resistance

Address correspondence and reprint requests to: E. De Rossi, Dipartimento di Genetica e Microbiologia, Università degli Studi di Pavia, via Ferrata 1, 27100 Pavia, Italy. Phone: +39-0382-505575; fax: +39-0382-528496; e-mail: derossi@ipvgen.unipv.it mechanisms among microorganisms; it has been demonstrated to occur in many Gram-positive and Gram-negative bacteria including medically important species (3).

Bacterial drug efflux pumps proved to occur in five families (4). Two of these are large and ancient superfamilies known as the ATP binding cassette (ABC) superfamily and the major facilitator superfamily (MFS). The other three are smaller and more recently developing families, called the small multidrug resistance (SMR) family, the resistancenodulation-cell division (RND) family, and the multidrug and toxic compounds extrusion family (MATE). The MFS, SMR, RND, and MATE families are secondary transporters, typically energized by the proton motive force. In contrast, ATP is utilized as the energy donor for members of the ABC family of multidrug efflux pumps that are often considered primary transporters. Several drug efflux pumps have been identified or characterized in mycobacteria (5-11), belonging to three of the five families just described.

First, members of the MFS have been described in several mycobacterial species. In M. smegmatis, the predicted LfrA protein, conferring resistance to several fluoroquinolones (5) and the Tet(V) protein, conferring resistance to tetracycline (6), appear to 
have 14 and 11 hydrophobic membrane-spanning regions, respectively. The $M$. fortuitum Tap (7) and the $M$. bovis P55 (8) proteins confer resistance to aminoglycosides and tetracycline. In M. tuberculosis, the putative Tap protein (7) confers resistance to tetracycline, and the EfpA protein does not seem to confer any phenotype (9).

Second, the Mmr protein of M. tuberculosis appears to be a multidrug efflux pump with four transmembrane regions belonging to SMR family (10).

Third, one member of the $\mathrm{ABC}$ transporter superfamily involved in fluoroquinolone resistance has been characterized in M. smegmatis (11). Recently, Braibant et al. (12), by inventory and assembly of the typical subunits of the ABC transporters encoded by the complete genome of $M$. tuberculosis, found that the genes encoding these proteins potentially implicated in the formation of the permeases account for $2.5 \%$ of the genome.

The MFS is one of the two largest families of membrane transporters. Compounds transported by MFS permeases include simple sugars, oligosaccharides, inositols, drugs, amino acids, nucleosides, organophosphate esters, Krebs cycle metabolites, and a large variety of inorganic anions and cations. They are found ubiquitously in all three domains of living organisms-bacteria, archaea, and eukaryotes $(13,14)$. Transport proteins of the MFS contain a number of conserved amino acid sequence motifs that are either ubiquitous within the MFS or subfamily specific (15). Conservation of such motifs among proteins responsible for the transport of a wide variety of structurally disparate compounds implies that they play some vital structural or functional role rather than directly interacting with their substrate(s). In particular, Paulsen et al. (16) identified motifs conserved throughout the MFS [motifs A (GxLaDrxGrkxxxl) and B (lxxxRxxqGxgaa)]; motif C (gxxxGPxxGGxl), also known as the antiporter motif, can be found in TMS 5 of the drug/proton antiporters of either 12- or 14-TMS, but not in symporters from other MFS subfamilies, suggesting that it may be required for linking proton translocation to antiport but not to symport of a substrate $(16,17)$; other motifs are exclusive to either the 12- or 14-TMS subfamily (motifs D to $\mathrm{H}$ ).

The importance of bacterial MFS transporters in conferring drug resistance is generally accepted. In mycobacteria, the majority of drug efflux pumps characterized so far belongs to the MFS, which reinforces the importance of this family of transporters in the innate drug resistance of the tubercle bacillus. Thus, we decided to analyze the MFS transporters encoded by the $M$. tuberculosis genome (18), which may be potentially involved in antibiotic extrusion.

In this study, we applied procedures, based on the bioinformatic tools, to identify and characterize drug efflux proteins belonging to MFS in M. tuberculosis. To investigate the power of prediction and the substrate specificity of potential drug transporters, we cloned 11 putative drug transporter genes of M. tuberculosis into different plasmids and then investigated their drug resistance phenotypes in $M$. smegmatis. In the case of two genes, we also demonstrated that they function as drug efflux proteins.

Data reported could be useful for the planning of further experimental work, necessary for the physiological characterization of these transporters and for the conception of new therapeutic agents.

\section{Material and Methods Computer Methods}

This analysis integrates different approaches and is based on the conservation of three features between drug transporters in microorganisms: specific sequence motifs, overall sequence similarity, and structural similarities (transmembrane segments). The $M$. tuberculosis genome sequence was retrieved from the Tuberculist web server at Institut Pasteur (http://genolist.pasteur.fr/TubercuList) (18).

Searches of the stretches of amino acids containing the specific motifs conserved in and characteristic of members of the MFS among the M. tuberculosis proteins was done using the SEARCH PATTERN program provided at the Tuberculist web server. The MEME program (http://www.sdc.edu/MEME) (19) was also used in identifying conserved motifs within the protein families. To well characterize the specific motifs present in the M. tuberculosis transporters, the regions containing the motifs were aligned using the CLUSTALW program (http://www.ebi.ac.uk) (20). CLUSTALW was used also to generate the phylogenetic tree, and TreeView (21) was used to display it. Searches of sequence similarity between potential drug transporters and proteins characterized in other organisms, were done using the BLAST program (22). BLAST searches of the M. tuberculosis proteins were performed at the Sanger Centre web page (http://www.sanger.ac.uk/Projects/M_tuberculosis/ blast_server.shtml). This allowed for the identification of additional putative transporters encoded in the $M$. tuberculosis genome. Also, proteins encoded in the $M$. smegmatis genome were searched at the BLAST Server of The Institute for Genomic Research (http://tigrblast.tigr.org/ufmg/index.cgi?database= m_smegmatislseq).

The number and the position of the transmembrane domains have been predicted by TMHMM (http://www.cbs.dtu.dk/services/TMHMM-2.0) (23).

\section{Strains and Growth Conditions}

Escherichia coli XL1-Blue, used as the cloning host, was grown in LB medium. M. tuberculosis H37Rv was used as the donor of chromosomal DNA. M. smegmatis $\mathrm{mc}^{2} 155$, selected to test the drug susceptibility, was grown in Middlebrook $7 \mathrm{H} 9$ broth and Middlebrook $7 \mathrm{Hll}$ agar (Difco) supplemented with $10 \%$ Middlebrook OADC enrichment (Difco) and $0.2 \%$ glycerol. All the cultures were incubated at $37^{\circ} \mathrm{C}$. 
Kanamycin was added, when required, at final concentrations of 50 and $25 \mu \mathrm{g} / \mathrm{ml}$ for E. coli and M. smegmatis, respectively.

\section{Cloning Procedures}

Genes predicted to encode hypothetical drug efflux pumps were amplified by polymerase chain reaction (PCR) from M. tuberculosis H37Rv chromosomal DNA using specific primers containing the restriction enzyme sites useful for subsequent cloning. ORFs Rv0037c, Rv0849, Rv1877, and Rv2994 were cloned in pMD31 (24) and pSUM38 (25) vectors. Similarly, ORFs Rv0783c, Rv1250, Rv2333c, Rv2559, and Rv3239c were cloned in pSUM38, and ORF Rv1258c was cloned in pSUM36 (25). Rv1634 was cloned directly from cosmid Z95554 digested with KpnI. Each cloned ORF contained about 1000 bp upstream the start translation codon to ensure the presence of native promoter. Plasmid DNA was isolated from E. coli with Qiagen columns prior to transformation of M. smegmatis by electroporation.

\section{Drug Susceptibility Test}

Minimal inhibitory concentrations (MIC) of drugs were determined on 7H11 medium supplemented with OADC and containing various drugs (chloramphenicol, tetracycline, ciprofloxacin, lomefloxacin, isoniazid, ethambutol, rifampicin, erythromycin, ethidium bromide, rhodamine 123, doxorubicin, gentamicin, streptomycin, and 2'-N- and $6^{\prime}-\mathrm{N}$-ethylnetilmicin) at various concentrations as indicated. Plates were inoculated with a total of $10^{4}$ cells and incubated at $37{ }^{\circ} \mathrm{C}$ for $4-6$ days and then growth was evaluated.

\section{Measurement of Norfloxacin Accumulation}

The modified fluorimetric method of Mortimer and Piddock (26) was altered slightly to accommodate the growth characteristics of the mycobacteria, as described by Williams et al. (27).

\section{Tetracycline Accumulation Assays}

The accumulation of titriated tetracycline in M. smegmatis cells was monitored as described previously $(7,8)$.

\section{Results and Discussion}

Analysis of MFS Drug Transporters ORFs

To identify the $M$. tuberculosis drug efflux proteins belonging to MFS, we first scanned the M. tuberculosis predicted proteins for the presence of the transporter family signature motifs A and B, using the program SEARCH PATTERN at the TubercuList web server. In motifs A (GXL[AG]D[RI]XGX[KR]XX[LF][LI]) and B (L[VL]XXRXX[QLA]GXG[AG][AG]), X represents any amino acid, and residues in brackets indicate those most frequently observed in a single position in more than $75 \%$ of transport proteins. Allowing none or one mismatch in the $\mathrm{A}$ and $\mathrm{B}$ transporter family signatures, no proteins were identified. By increasing progressively the number of mismatches to four in motifs A and B, 540 potential motifs in 290 different proteins were identified. Because, in this superfamily, motifs A and B are separated by 18-30 amino acids, we checked if the identified proteins contained each of these two motifs at a correct relative position. If not, they were discarded.

The proteins in which motifs $\mathrm{A}$ and $\mathrm{B}$ were properly located were subjected to TMHMM to analyze the transmembrane profile; because MFS proteins are characterized by more than 12-TMS, we considered initially the following 19 ORFs: Rv0102 with 16-TMS, Rv3239c and Rv3728 with 15-TMS, Rv2846c, Rv1877, Rv2333c, Rv2459, Rv0783c, Rv1634, Rv1250, Rv1410c with 14-TMS, and Rv1258c, Rv0849, Rv0842, Rv1217c, Rv1510, DinF, NarK1, NarK2, and SugI with 12-TMS.

BLASTP analysis of these proteins confirmed they all belong to the MFS. However, this analysis also revealed that only 12 of them (Rv3239c, Rv3728, Rv2846c, Rv1877, Rv2333c， Rv2459, Rv1410c, Rv1250, Rv1258c, Rv0783c, Rv1634, and Rv0849) showed sequence similarity to drug efflux pumps, previously characterized in other bacteria. As shown in Figure 1, motifs A and B, typical of MFS transporters, are present. Further, as expected, motif $\mathrm{C}$, typical of drug antiporters, could be found in these twelve proteins, as revealed by Clustalw alignment (Fig. 1).

Proteins Rv3728 and Rv3239c are unusually long for being drug transporters; they have 1065 and 1048 amino acids, respectively. The sequence similarity with multidrug efflux transporters and the position of the transmembrane segments is restricted to the first 500-600 residues. However, they were also considered because they showed the MFS motifs A, B, and C clearly conserved and properly located (Fig. 1).

By ClustalW alignment of the ORFs with 14 and 15 TMS, motifs D $1, \mathrm{H}, \mathrm{E}$, and F were identified (Fig. 2A); in the 12 TMS ORFs, motif $G$ has been identified (Fig. 2B).

To identify possible additional drug efflux proteins not selected by the strategy mentioned, a search of sequence similarity (BLASTP) within all proteins of $M$. tuberculosis H37Rv was done using each of the 12 proteins previously identified as a query. This search allowed the identification of four additional hypothetical efflux pumps: Rv0191, Rv0037c, Rv2456c, and Rv2994. ClustalW alignment of these hypothetical efflux pumps revealed the presence of motifs A and B (data not shown), indicating their belonging to MFS superfamily; concerning motif $\mathrm{C}$, typical of the drug efflux transporters, a possible consensus sequence was identified, although not well conserved (data not shown). Interestingly, Rv0037c, Rv2456c, and Rv2994 were retrieved among the first set of 290 proteins containing motifs $\mathrm{A}$ and $\mathrm{B}$ that were 


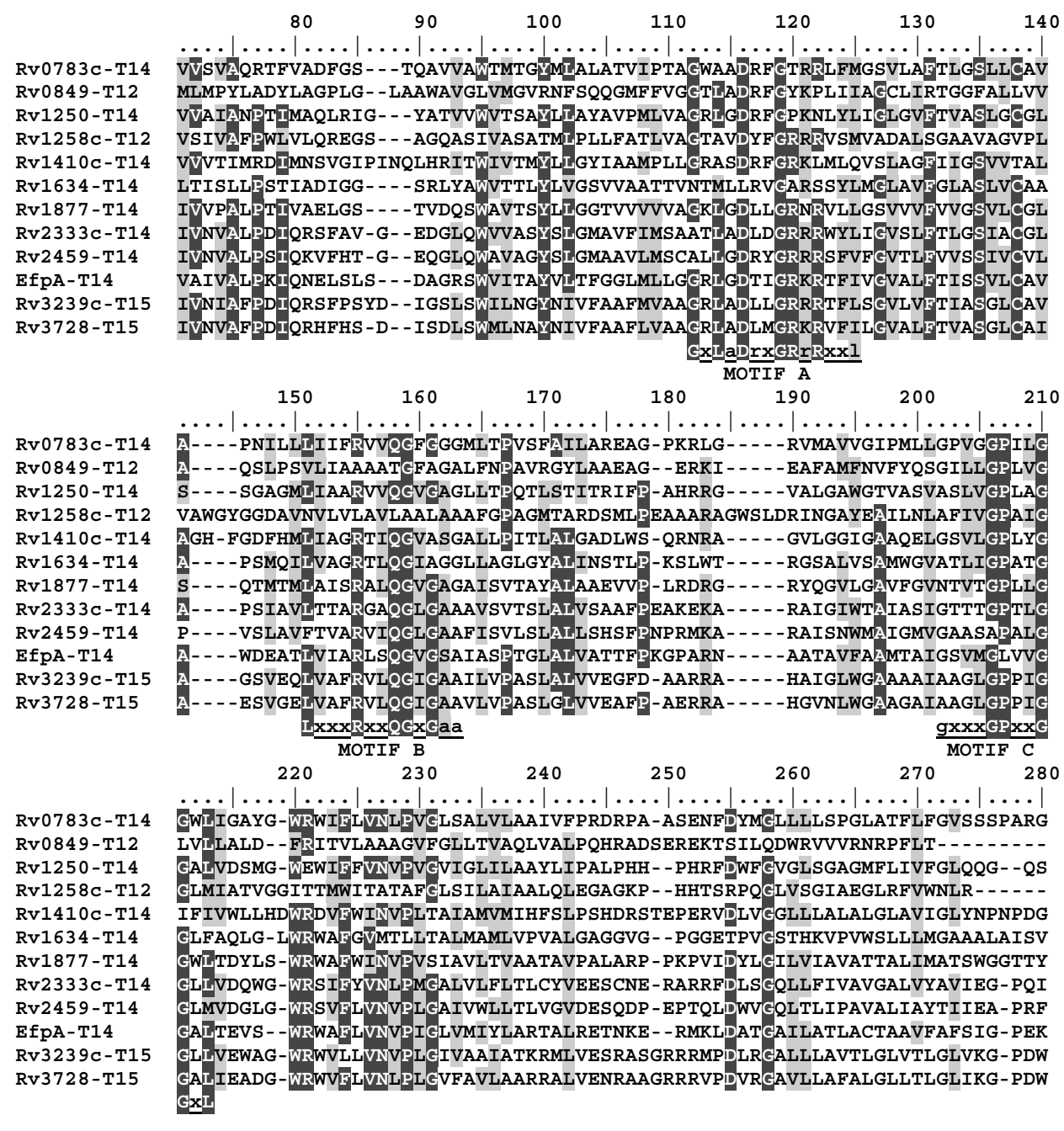

Fig. 1. Multiple-sequence alignment for 12 members of the hypothetical mycobacterial drug efflux proteins. Highly conserved motifs are displayed below the alignment; the consensus sequences of the motifs are displayed as follows: $\mathrm{x}$, any amino acids; capital letters, the frequency of occurrence of the amino acids is greater that $70 \%$; lower case letters, the frequency of occurrence of the amino acids is greater than $40 \%$. Motifs A, B, and C correspond to the motifs described by Paulsen et al. (16). The alignment shows only the region containing the above-cited motifs. Identical amino acids are highlighted in black boxes; conserved amino acid substitutions are shown in shaded boxes. identified by using the SEARCH PATTERN program, but they were not considered for further analysis because their TMS numbers were 11,10 , and 9, respectively. This could be indicate that transporters with a number of TMS smaller than 12 may be also involved in drug transport. Rv0191 is predicted to contain 12 TMS; however, this protein was not selected because of the high number of mismatches between its motifs A and B and those used for the screening.

In summary, from our analysis, we retrieved a total of 16 hypothetical efflux proteins, whose characteristics are described in Table 1. In these 16 protein sequences, motifs $\mathrm{A}, \mathrm{B}$, and $\mathrm{C}$ are highly conserved, independent of the number of TMS. This has allowed us to define more precisely the sequence motifs A, B, and C, specifically for the $M$. tuberculosis drug efflux proteins (Tables $2 \mathrm{~A}, 2 \mathrm{~B}$, and 2C, respectively). In this way, motif $\mathrm{A}$ in M. tuberculosis can be regarded as GrLaDrfGrRRv[l,f]l (versus general consensus GxLaDrxGrkxxxl); motif $B$ would be LvaaRvlQGxGA[g,a] (versus general consensus lxxxRxxqGxgaa); finally, motif $\mathrm{C}$, which is highly conserved in the majority of drug efflux proteins, can be defined as gxvxGPllGGlL (versus general consensus gxxxGPxxGGxl). These signatures will be very useful in identifying and characterizing new members of the drug efflux family from other mycobacterial species. For example, in M. smegmatis two drug transporters of the MFS superfamily have been identified, LfrA and Tet(V), which do not seem to have any homologue in the M. tuberculosis chromosome. This indicates that $M$. smegmatis (for which it has been estimated a genome size of 7.5 Mb versus the $4.4 \mathrm{Mb}$ of $M$. tuberculosis) and also other mycobacterial species will contain many other MFS transporters, different from those identified in $M$. tuberculosis.

From the CLUSTALW alignment of these transporters, a phylogenetic tree showing the relationships among the proteins is shown in Figure 3. It appears that the proteins potentially involved in M. tuberculosis drug efflux are scattered in three different groups. Two of the three groups include proteins with 14-15 transmembrane segments; the third group is composed by a subgroup of proteins 


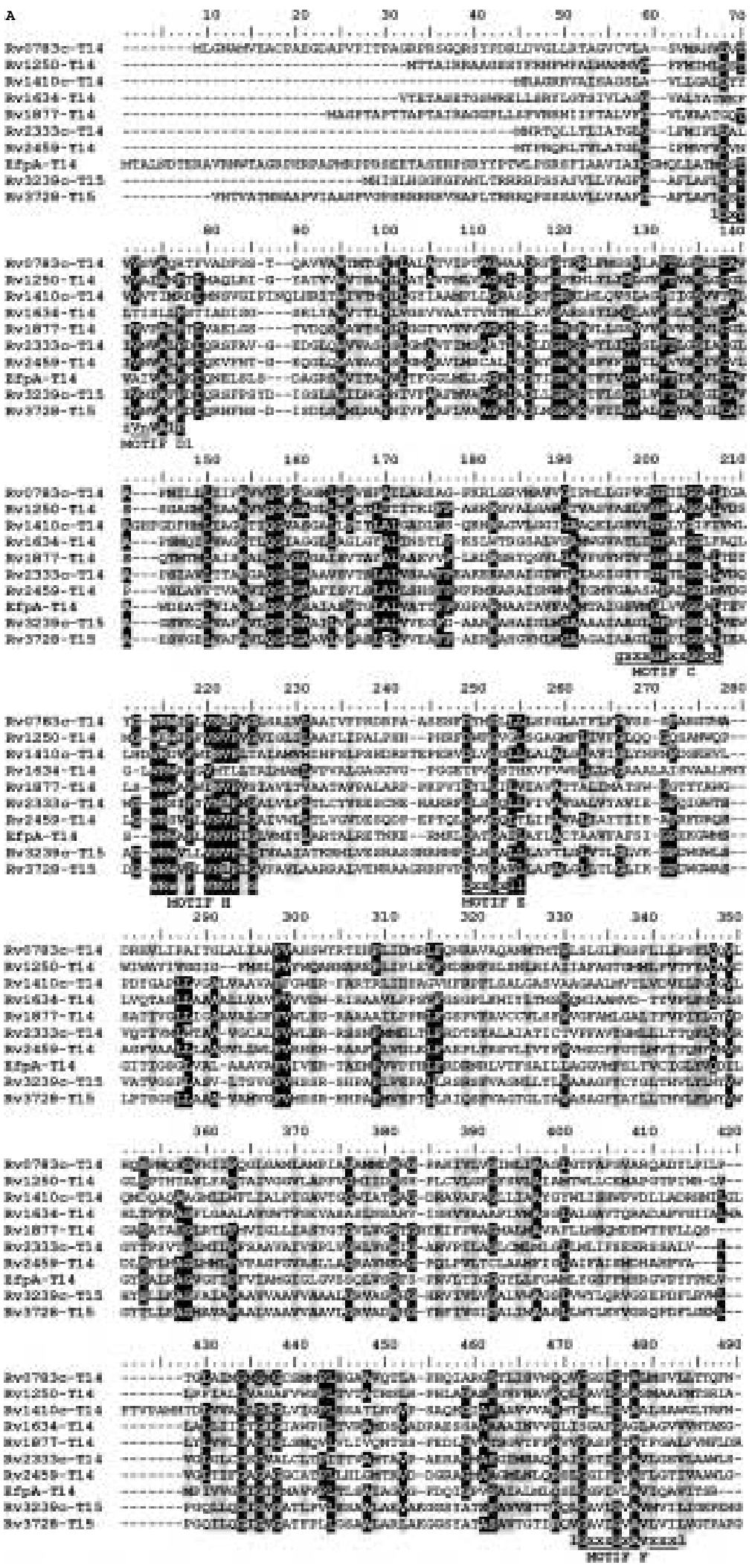

Fig. 2. Multiple-sequence alignments for members of the mycobacterial 14- and 15-TMS (A), and 12-TMS drug efflux transporters (B), respectively. Details on these alignments can be found in the legend for Figure 1. Motifs D l, H, E, F, and G correspond to the motifs described by Paulsen et al. (16). Motif C, described as characteristic of drug export proteins, is indicated. The alignment shows only the region containing the above-cited motifs. Identical amino acids are highlighted in black boxes; conserved amino acid substitutions are shown in shaded boxes. 


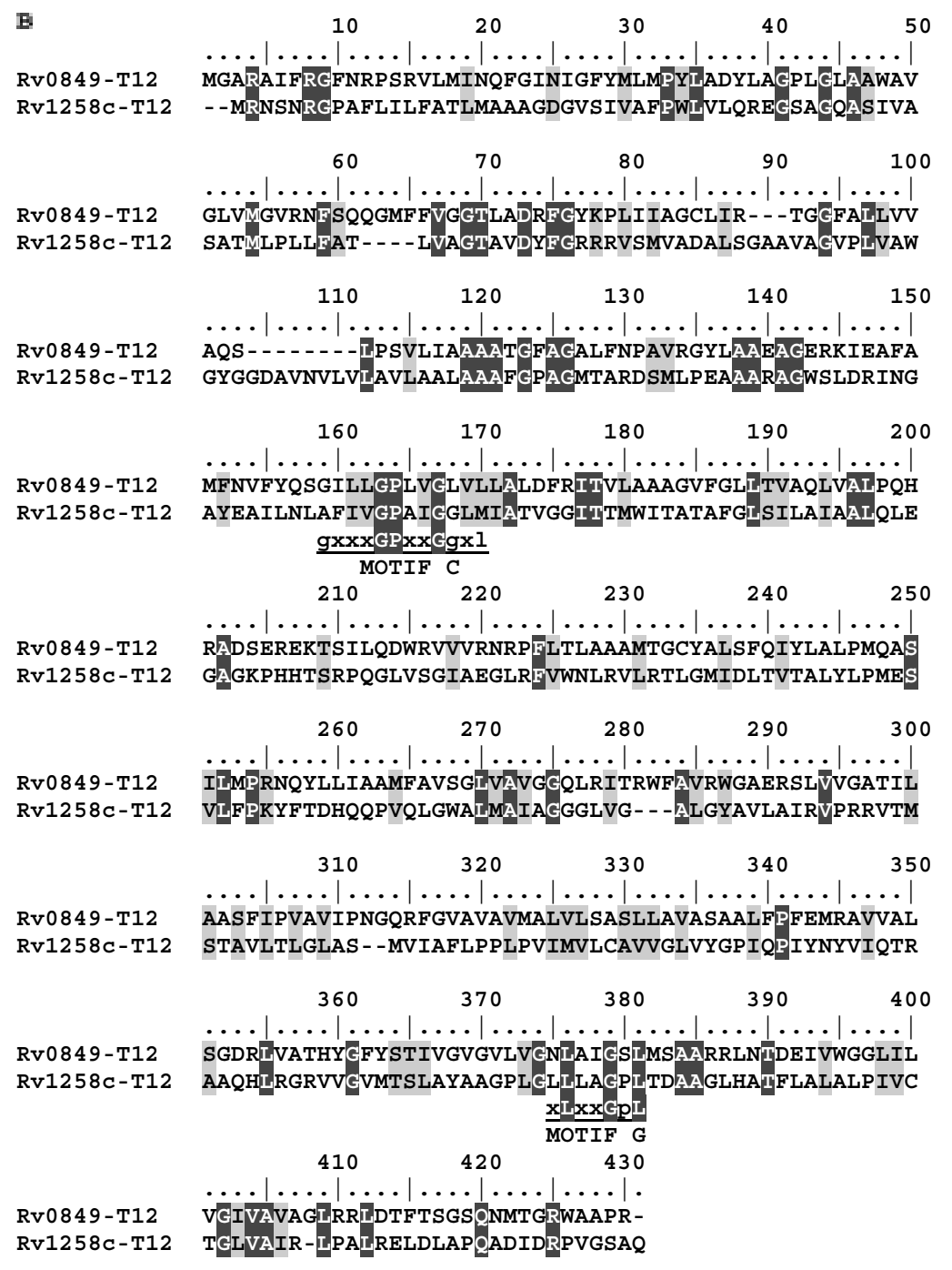

Fig. 2. (Continued).

with 14 transmembrane segments (Rv1410c and Rv 1877) and by a subgroup of proteins with 12 or less than 12 transmembrane segments, with the exception of Rv1634.

\section{Cloning and Phenotypic Analysis of Hypothetical Drug Transporters}

Although most of the proteins identified in this analysis remain uncharacterized, a few of them have been previously shown to function as multidrug transporters; Rv1410c protein was demonstrated to be an efflux pumps, conferring resistance to tetracycline and aminoglycosides (8), similarly to the M. fortuitum homolog of Rv1258c (7). In the latter report, the expression of the M. tuberculosis Rv1258c gene under the control of the $h s p 60$ promoter conferred resistance to only tetracycline (but see below for a revision on this phenotype). In the case of Rv2846c, it has been published that this protein does not confer any resistant phenotype to M. smegmatis (9).
We decided to further characterize some of the M. tuberculosis H37Rv putative drug transporters of the MFS identified in this report.

Plasmids containing ORF Rv1634 conferred resistance to different fluoroquinolones (ciprofloxacin, norfloxacin, ofloxacin, and lomefloxacin) in M. smegmatis (Table 3A), demonstrating that a novel fluoroquinolone-resistant determinant is present in $M$. tuberculosis. The first efflux pump described in mycobacteria, LfrA, was identified in M. smegmatis because its overexpression conferred resistance to ciprofloxacin. LfrA belongs to the family of proton antiporters and, in addition to the hydrophilic fluoroquinolones, its overexpression also confers resistance to acriflavine and ethidium bromide (5). Disruption of the lfrA gene in $M$. smegmatis caused an increased sensitivity to ethidium bromide and acriflavine, but did not significantly affect resistance to ciprofloxacin (28), arguing against a major role of the LfrA in the innate resistance of $M$. smegmatis to 
Table 1. List of M. tuberculosis ORFs described as hypothetical drug efflux proteins belonging to MFS.

\begin{tabular}{|c|c|c|c|c|c|c|}
\hline \multirow[b]{2}{*}{ Sequence } & \multirow[b]{2}{*}{ Length } & \multirow[b]{2}{*}{ Hypothetical Function* } & \multirow{2}{*}{$\begin{array}{c}\text { TMS } \\
(N)\end{array}$} & \multicolumn{3}{|c|}{ Relative Position of } \\
\hline & & & & Motif A & Motif B & Motif C \\
\hline Rv0037c & 441 & Probable membrane protein & 11 & $89-101$ & $124-136$ & $169-180$ \\
\hline Rv0191 & 413 & Probable chloramphenicol resistance protein & 12 & $77-89$ & $111-123$ & $157-168$ \\
\hline Rv0783c & 540 & Multidrug resistance protein & 14 & $99-111$ & $134-146$ & $179-190$ \\
\hline Rv0849 & 419 & Possible quinolone efflux pump & 12 & $69-81$ & $104-116$ & $148-159$ \\
\hline Rv 1250 & 579 & Probable drug efflux protein & 14 & $75-87$ & $110-122$ & $152-163$ \\
\hline Rv1258c & 419 & Probable multidrug resistance pump & 12 & $63-75$ & $102-114$ & $153-164$ \\
\hline Rv1410c & 518 & Probable drug efflux protein & 14 & $66-78$ & $104-116$ & $149-160$ \\
\hline Rv1634 & 471 & Probable drug efflux protein & 14 & $75-87$ & $110-122$ & $155-166$ \\
\hline Rv1877 & 687 & Probable drug efflux protein & 14 & $83-95$ & $108-120$ & $163-174$ \\
\hline Rv2333c & 537 & Probable tetracenomycin C resistance protein & 14 & $63-75$ & $98-110$ & $145-156$ \\
\hline Rv2456c & 418 & Probable transmembrane transport protein & 10 & $68-80$ & $104-116$ & $149-160$ \\
\hline Rv2459 & 508 & Probable drug efflux protein & 14 & $63-75$ & $98-110$ & $145-156$ \\
\hline $\begin{array}{l}\text { Rv2846c } \\
\text { (EfpA) }\end{array}$ & 530 & EfpA putative efflux protein & 14 & $109-121$ & $144-156$ & $190-201$ \\
\hline Rv2994 & 445 & Probable fluoroquinolone efflux protein & 9 & $69-81$ & $104-116$ & $149-160$ \\
\hline Rv3239c & 1048 & Probable drug efflux protein & 15 & $81-93$ & $116-128$ & $161-172$ \\
\hline Rv3728 & 1065 & Possible sugar transporter & 15 & $97-109$ & $132-144$ & $177-188$ \\
\hline
\end{tabular}

*As reported by Cole et al. (18).

Table 2A. Common sequence motifs A localized in TMS2 and TMS3 in the mycobacterial drug efflux transporters.

\begin{tabular}{|c|c|c|c|c|c|c|c|c|c|c|c|c|c|}
\hline \multirow[b]{2}{*}{ Sequence } & \multicolumn{13}{|c|}{ Predominant Residue at Position } \\
\hline & $\begin{array}{l}\mathbf{1} \\
\mathbf{G}\end{array}$ & $\begin{array}{l}\mathbf{2} \\
\mathbf{X}\end{array}$ & $\begin{array}{l}3 \\
\mathbf{L}\end{array}$ & $\begin{array}{l}\mathbf{4} \\
\mathbf{A}\end{array}$ & $\begin{array}{l}5 \\
\text { D }\end{array}$ & $\begin{array}{l}6 \\
\mathbf{R}\end{array}$ & $\begin{array}{l}7 \\
\mathbf{X}\end{array}$ & $\begin{array}{l}\mathbf{8} \\
\mathbf{G}\end{array}$ & $\begin{array}{l}\mathbf{9} \\
\mathbf{R}\end{array}$ & $\begin{array}{l}10 \\
\text { K }\end{array}$ & $\begin{array}{l}11 \\
X\end{array}$ & $\begin{array}{l}12 \\
X\end{array}$ & $\begin{array}{c}13 \\
\mathbf{L}\end{array}$ \\
\hline Rv0037c & G & A & $\mathrm{L}$ & M & $\mathrm{D}$ & $\mathbf{R}$ & $\mathrm{W}$ & $\mathrm{D}$ & $\mathbf{R}$ & $\mathbf{R}$ & W & V & $\mathrm{L}$ \\
\hline Rv0191 & $\mathrm{V}$ & $\mathbf{R}$ & W & $\mathrm{T}$ & A & $\mathrm{H}$ & $\mathrm{W}$ & $\mathrm{P}$ & $\mathbf{R}$ & $\mathbf{R}$ & $\mathbf{R}$ & A & $\mathrm{L}$ \\
\hline Rv0783c & G & W & A & A & $\mathrm{D}$ & $\mathbf{R}$ & $\mathrm{F}$ & G & $\mathrm{T}$ & $\mathbf{R}$ & $\mathbf{R}$ & $\mathrm{L}$ & $\mathrm{F}$ \\
\hline Rv0849 & G & $\mathrm{T}$ & $\mathrm{L}$ & A & $\mathrm{D}$ & $\mathbf{R}$ & $\mathrm{F}$ & G & $\mathrm{Y}$ & K & $\mathrm{P}$ & $\mathrm{L}$ & I \\
\hline Rv1250 & G & $\mathbf{R}$ & $\mathrm{L}$ & G & D & $\mathbf{R}$ & $\mathrm{F}$ & G & $\mathrm{P}$ & K & $\mathbf{N}$ & $\mathrm{L}$ & $\mathrm{Y}$ \\
\hline Rv1258c & G & $\mathrm{T}$ & A & $\mathrm{V}$ & D & $\mathrm{Y}$ & $\mathrm{F}$ & G & $\mathbf{R}$ & $\mathbf{R}$ & $\mathbf{R}$ & $\mathrm{V}$ & $S$ \\
\hline Rv1410c & G & $\mathbf{R}$ & A & $\mathrm{S}$ & D & $\mathbf{R}$ & $\mathrm{F}$ & G & $\mathbf{R}$ & $\mathrm{K}$ & $\mathrm{L}$ & $\mathbf{M}$ & $\mathrm{L}$ \\
\hline Rv1634 & $\mathrm{N}$ & $\mathrm{T}$ & $\mathbf{M}$ & $\mathrm{L}$ & $\mathrm{L}$ & $\mathbf{R}$ & $\mathrm{V}$ & G & A & $\mathbf{R}$ & $S$ & $S$ & $\mathrm{Y}$ \\
\hline Rv 1877 & G & $\mathrm{K}$ & L & G & D & $\mathrm{L}$ & $\mathrm{L}$ & G & $\mathbf{R}$ & $\mathrm{N}$ & $\mathbf{R}$ & $\mathrm{V}$ & $\mathrm{L}$ \\
\hline Rv2333c & A & $\mathrm{T}$ & $\mathrm{L}$ & A & $\mathrm{D}$ & $\mathrm{L}$ & $\mathrm{D}$ & G & $\mathbf{R}$ & $\mathbf{R}$ & $\mathbf{R}$ & $\mathrm{W}$ & $\mathrm{Y}$ \\
\hline Rv2456c & G & A & $\mathrm{L}$ & $\mathrm{V}$ & $\mathrm{D}$ & $\mathbf{R}$ & $\mathrm{T}$ & $\mathrm{T}$ & A & K & $\mathbf{R}$ & A & $\mathrm{L}$ \\
\hline Rv2459 & A & $\mathrm{L}$ & $\mathrm{L}$ & G & $\mathrm{D}$ & $\mathbf{R}$ & $\mathrm{Y}$ & G & $\mathrm{R}$ & $\mathbf{R}$ & $\mathbf{R}$ & $S$ & $\mathrm{~F}$ \\
\hline Rv2846c & G & $\mathbf{R}$ & $\mathrm{L}$ & G & $\mathrm{D}$ & $\mathrm{T}$ & I & G & $\mathrm{R}$ & K & $\mathbf{R}$ & $\mathrm{T}$ & $\mathrm{F}$ \\
\hline Rv2994 & G & $\mathrm{Y}$ & $\mathrm{L}$ & $\mathrm{L}$ & $\mathrm{D}$ & $\mathrm{H}$ & $\mathrm{V}$ & G & $\mathrm{E}$ & $\mathbf{R}$ & M & $\mathrm{V}$ & M \\
\hline Rv3239c & G & $\mathrm{R}$ & $\mathrm{L}$ & A & $\mathrm{D}$ & $\mathrm{L}$ & $\mathrm{L}$ & G & $\mathbf{R}$ & $\mathbf{R}$ & $\mathbf{R}$ & $\mathrm{T}$ & $\mathrm{F}$ \\
\hline Rv3728 & G & $\mathrm{R}$ & $\mathrm{L}$ & A & $\mathrm{D}$ & $\mathrm{L}$ & M & G & $\mathbf{R}$ & $\mathrm{K}$ & $\mathbf{R}$ & V & $\mathrm{F}$ \\
\hline Consensus & G & $\mathbf{R}$ & $\mathrm{L}$ & {$[\mathrm{GA}]$} & $\mathrm{D}$ & $\mathbf{R}$ & $\mathrm{F}$ & G & $\mathbf{R}$ & {$[\mathrm{RK}]$} & $\mathbf{R}$ & $\mathrm{V}$ & {$[\mathrm{LF}]$} \\
\hline
\end{tabular}


Table 2B. Common sequence motifs B localized in TMS4 in the mycobacterial drug efflux transporters.

Predominant Residue at Position

\begin{tabular}{llllllllllllll} 
Sequence & I & $\mathbf{2}$ & $\mathbf{3}$ & $\mathbf{4}$ & $\mathbf{5}$ & $\mathbf{6}$ & $\mathbf{7}$ & $\mathbf{8}$ & $\mathbf{9}$ & $\mathbf{1 0}$ & $\mathbf{1 1}$ & $\mathbf{1 2}$ & $\mathbf{1 3}$ \\
\hline Rv0037c & G & D & V & P & L & L & V & G & A & L & V & A & N \\
Rv0191 & L & A & A & G & R & V & L & C & A & V & T & H & G \\
Rv0783c & L & I & I & F & R & V & V & Q & G & F & G & G & G \\
Rv0849 & V & L & I & A & A & A & A & T & G & F & A & G & A \\
Rv1250 & L & I & A & A & R & V & V & Q & G & V & G & A & G \\
Rv1258c & N & V & L & V & L & A & V & L & A & A & L & A & A \\
Rv1410c & L & I & A & G & R & T & I & Q & G & V & A & S & G \\
Rv1634 & L & V & A & G & R & T & L & Q & G & I & A & G & G \\
Rv1877 & L & A & I & S & R & A & L & Q & G & V & G & A & G \\
Rv233c & L & T & T & A & R & G & A & Q & G & L & G & A & A \\
Rv2456c & I & S & V & L & Q & A & V & T & G & I & A & S & S \\
Rv2459 & F & T & V & A & R & V & I & Q & G & L & G & A & A \\
Rv2846c & L & V & I & A & R & L & S & Q & G & V & G & S & A \\
Rv2994 & I & G & V & F & L & F & L & G & G & M & A & A & G \\
Rv3239c & L & V & A & F & R & V & L & Q & G & I & G & A & A \\
Rv3728 & L & V & A & F & R & V & L & Q & G & I & G & A & A \\
Consensus & L & V & A & A & R & [VA] & [LV] & Q & G & [VI] & G & A & [GA]
\end{tabular}

Table 2C. Common sequence motifs C localized in TMS5 in the mycobacterial drug efflux transporters.

\begin{tabular}{|c|c|c|c|c|c|c|c|c|c|c|c|c|}
\hline \multirow[b]{2}{*}{ Sequence } & \multicolumn{12}{|c|}{ Predominant Residue at Position } \\
\hline & $\begin{array}{l}\mathbf{I} \\
\mathbf{G}\end{array}$ & $\begin{array}{l}\mathbf{2} \\
\mathbf{X}\end{array}$ & $\begin{array}{l}\mathbf{3} \\
\mathbf{X}\end{array}$ & $\begin{array}{l}4 \\
X\end{array}$ & $\begin{array}{l}5 \\
\mathbf{G}\end{array}$ & $\begin{array}{l}6 \\
P\end{array}$ & $\begin{array}{l}\mathbf{7} \\
\mathbf{X}\end{array}$ & $\begin{array}{l}\mathbf{8} \\
\mathbf{X}\end{array}$ & $\begin{array}{l}9 \\
\text { G }\end{array}$ & $\begin{array}{c}10 \\
\text { G }\end{array}$ & $\begin{array}{l}11 \\
X\end{array}$ & $\begin{array}{c}12 \\
\mathrm{~L}\end{array}$ \\
\hline Rv0037c & $\mathrm{S}$ & G & A & $\mathrm{V}$ & $\mathrm{S}$ & A & $\mathrm{F}$ & $\mathrm{L}$ & G & A & $\mathrm{N}$ & $\mathrm{F}$ \\
\hline Rv0191 & A & $\mathrm{L}$ & $\mathrm{V}$ & $\mathrm{V}$ & G & $\mathrm{S}$ & $\mathbf{P}$ & $\mathrm{L}$ & $\mathrm{T}$ & A & A & M \\
\hline Rv0783c & G & $\mathbf{P}$ & $\mathrm{V}$ & G & G & $\mathbf{P}$ & I & $\mathrm{L}$ & G & G & $\mathrm{W}$ & $\mathrm{L}$ \\
\hline Rv0849 & G & I & $\mathrm{L}$ & $\mathrm{L}$ & G & $\mathbf{P}$ & $\mathrm{L}$ & $\mathrm{V}$ & G & $\mathrm{L}$ & $\mathrm{V}$ & $\mathrm{L}$ \\
\hline Rv 1250 & A & $\mathrm{S}$ & $\mathrm{L}$ & $\mathrm{V}$ & G & $\mathbf{P}$ & $\mathrm{L}$ & A & G & G & A & $\mathrm{L}$ \\
\hline Rv1258c & A & F & I & $\mathrm{V}$ & G & $\mathrm{P}$ & A & I & G & G & $\mathrm{L}$ & M \\
\hline Rv1410c & G & $S$ & $\mathrm{~V}$ & $\mathrm{~L}$ & G & $\mathrm{P}$ & $\mathrm{L}$ & $\mathrm{Y}$ & G & I & F & I \\
\hline Rv1634 & A & $\mathrm{T}$ & $\mathrm{L}$ & I & G & $\mathrm{P}$ & A & $\mathrm{T}$ & G & G & $\mathrm{L}$ & $\mathrm{F}$ \\
\hline Rv1877 & $\mathbf{N}$ & $\mathrm{T}$ & $\mathrm{V}$ & $\mathrm{T}$ & G & $\mathbf{P}$ & $\mathrm{L}$ & $\mathrm{L}$ & G & G & $\mathrm{W}$ & $\mathrm{L}$ \\
\hline Rv2333c & G & $\mathrm{T}$ & $\mathrm{T}$ & $\mathrm{T}$ & G & $\mathrm{P}$ & $\mathrm{T}$ & $\mathrm{L}$ & G & G & $\mathrm{L}$ & $\mathrm{L}$ \\
\hline Rv2456c & G & $\mathrm{N}$ & A & $S$ & A & A & G & A & $\mathrm{T}$ & G & A & $\mathrm{L}$ \\
\hline Rv2459 & G & A & A & $S$ & A & $P$ & A & $\mathrm{L}$ & G & G & $\mathrm{L}$ & M \\
\hline Rv2846c & G & $S$ & $\mathrm{~V}$ & M & G & $\mathrm{L}$ & V & V & G & G & A & $\mathrm{L}$ \\
\hline Rv2994 & G & I & A & $S$ & G & A & $\mathrm{L}$ & V & I & $P$ & E & $\mathrm{L}$ \\
\hline Rv3239c & A & A & G & $\mathrm{L}$ & G & $\mathrm{P}$ & $\mathrm{P}$ & I & G & G & $\mathrm{L}$ & $\mathrm{L}$ \\
\hline Rv3728 & $\mathrm{A}$ & A & G & $\mathrm{L}$ & G & $\mathrm{P}$ & $\mathrm{P}$ & I & G & G & A & $\mathrm{L}$ \\
\hline Consensus & {$[\mathrm{GA}]$} & [TA] & $\mathrm{V}$ & [VL] & G & $\mathrm{P}$ & $\mathrm{L}$ & $\mathrm{L}$ & G & G & [AL] & $\mathrm{L}$ \\
\hline
\end{tabular}




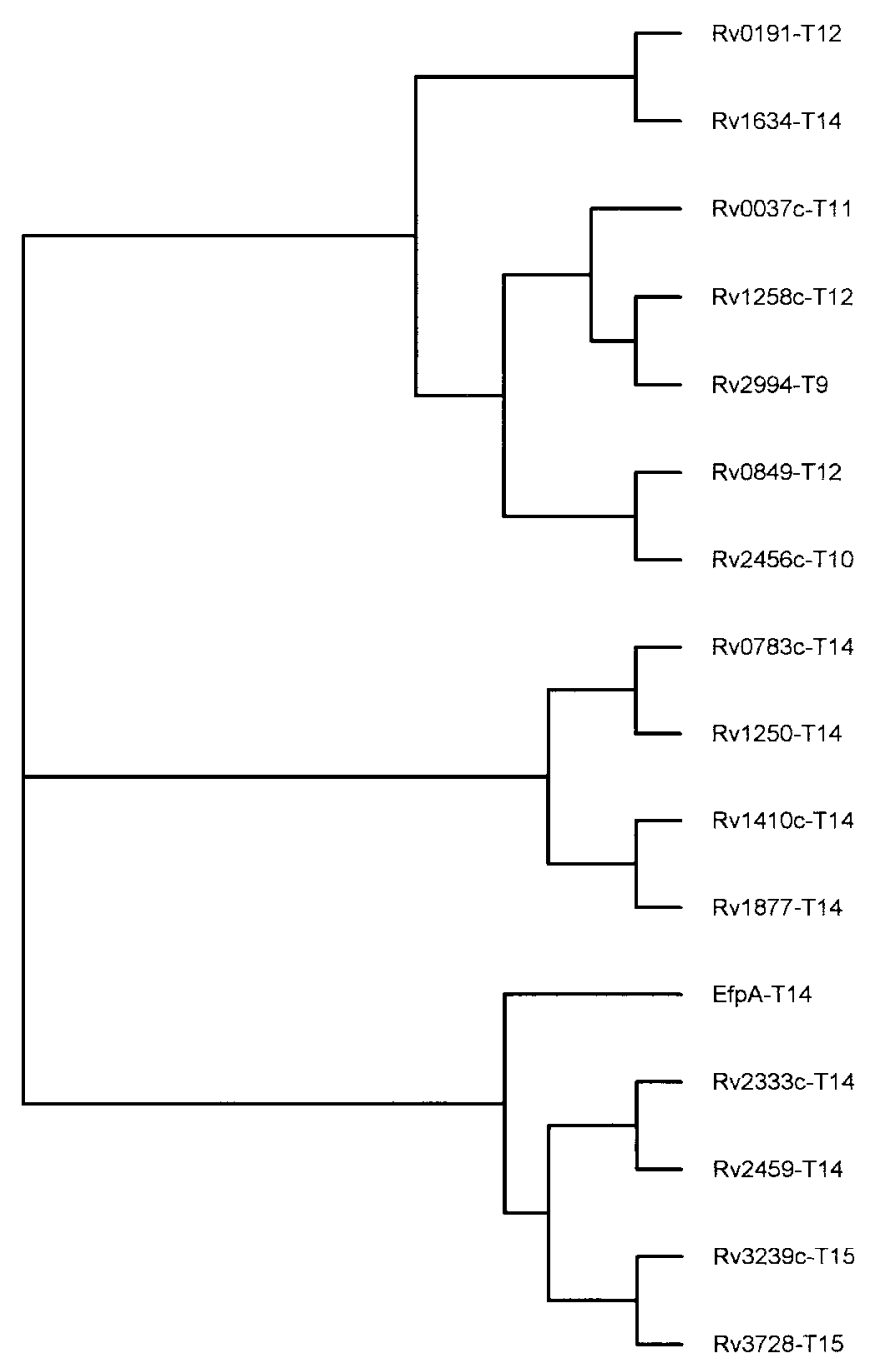

Fig. 3. Phylogenetic tree showing the relationships among the drug transporters subfamily of $M$. tuberculosis belonging to the MFS.

this drug, and suggesting the involvement of additional quinolone transporters in this species. As mentioned, homologs of the M. smegmatis lfrA gene could not be detected in the $M$. tuberculosis genome; however, we have detected a gene highly homologous to Rvl634 on the M. smegmatis chromosome (data not shown). Consequently, the M. smegmatis homologue of Rvl634 could be the real transporter responsible for fluoroquinolones efflux. The M. leprae genome contains a nucleotide sequence similar to ORF1634, but no corresponding protein was found in the Leproma website (http://genolist.pasteur.fr/Leproma), suggesting that it may be a pseudogene.

As mentioned, ORF Rv1258c expressed from the hsp60 promoter conferred resistance to only tetracycline, whereas its $M$. fortuitum homolog also conferred resistance to several aminoglycosides (7). As a part of this work, we decided to make a new construct containing the $M$. tuberculosis Rv1258c and the region upstream of it, where its own promoter should be located. The new plasmid was introduced in M. smegmatis and it conferred detectable levels of resistance to tetracycline and aminoglycosides (Table 3B). The $M$. tuberculosis putative efflux pump Rv1258c transports the same antibiotics that its M. fortuitum homolog, although the levels of resistance conferred by the M. tuberculosis protein are notably lower.

ORFs Rv0037c, Rv0783c, Rv0849， Rv1250, Rv1877, Rv2333c, Rv2459, Rv2994, and Rv3239c were amplified by PCR, cloned into different vectors, and transformed into M. smegmatis. MICs of a broad range of compounds (including representative cationic dyes, antimicrobial agents, antiseptics, anticancer drugs, uncouplers, and detergents) were determined for $M$. smegmatis cells harboring these plasmids.

Apparently, none of these genes confer resistance to any of the compounds tested at detectable levels. It is conceivable that either we have not tested the real substrates of these efflux pumps or that the resistance levels are so low that we are unable to detect them using our methodology. Another possibility is that the clones we analyzed do not contain the sequences necessary for its expression. Also, we cannot rule out the possibility that $M$. smegmatis transcription machinery is unable to express some M. tuberculosis genes. Further experiments are being carried out to elucidate the substrate profile and the role of these putative efflux pumps.

Table 3A. Drug resistance of $M$. smegmatis cells harboring plasmids carrying putative drug transporter Rv1634.

\begin{tabular}{llccc}
\hline & \multicolumn{3}{c}{ Antibiotics $(\mu \mathrm{g} / \mathrm{ml})$} \\
\cline { 2 - 5 } mc $^{2}$ 155 Transformed with & Ciprofloxacin & Lomefloxacin & Norfloxacin & Ofloxacin \\
\hline pMD31 & 0.12 & 0.06 & 1.25 & 0.125 \\
pMD31/Rv1634 & 0.48 & 0.24 & 5.0 & 0.25 \\
pSUM38 & 0.12 & 0.06 & 1.25 & 0.125 \\
pSUM38/Rv1634 & 0.24 & 0.12 & 5.0 & 0.25 \\
\hline
\end{tabular}


Table 3B. Drug resistance of $M$. smegmatis cells harboring plasmids carrying putative drug transporter Rv1258c.

Antibiotics $(\mu \mathrm{g} / \mathrm{ml})$

$\mathrm{mc}^{2} 155$

Transformed with Tetracycline Streptomycin Gentamicin $\quad 2^{\prime}$-N-Ethylnetilmicin 6'-N-Ethylnetilmicin $^{\prime}$

pSUM36

pSUM36/Rv1258c

0.5

4

2
4

2

2
8

8

\section{Accumulation of Norfloxacin}

The resistance levels conferred to $M$. smegmatis by Rv1634, cloned into two different vectors, were 2- to 4-fold the MIC value for fluoroquinolones (Table 3A). In general, efflux pumps confer low levels of resistance, which contrast with the high levels conferred by mutations in genes encoding the primary targets of these agents (DNA topoisomerase and DNA gyrase) (29).

Norfloxacin uptake experiments were performed to determine whether $M$. smegmatis cells, carrying the recombinant plasmid pMD31/Rv1634, were more resistant to this drug due to an active drug efflux mechanism. The accumulation of norfloxacin was determined by fluorimetry, as described in Material and Methods.

As shown in Figure 4A, cells harboring the cloning vector pMD31 take up norfloxacin rapidly and achieve a steady-state level of accumulation within about 2-3 min of incubation. This accumu-

A

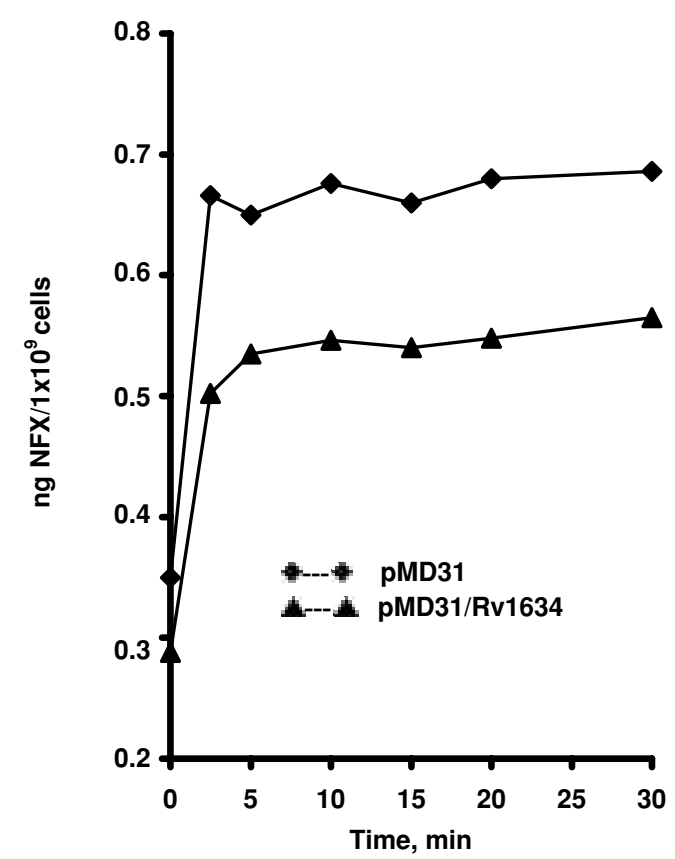

lation is approximately $25 \%$ lower with cells containing the pMD31/Rv1634 recombinant plasmid. The reduction of norfloxacin uptake was repeatedly observed in several experiments. Analogous results were obtained with ciprofloxacin (data not shown). These accumulation data suggest that Rv1634 could be involved in fluoroquinolones efflux.

\section{Accumulation of Tetracycline}

M. smegmatis transformed with a plasmid-borne Rv1258c gene showed an 8-fold increase in the resistance levels to tetracycline $(4 \mu \mathrm{g} / \mathrm{ml})$ in comparison with the same strain transformed with the vector alone $(0.4 \mu \mathrm{g} / \mathrm{ml})$ (Table $3 \mathrm{~B})$. We used radiolabeled tetracycline to measure whether this increase of resistance was due to a lower accumulation of this drug.

Accumulation assays showed that the presence of the plasmid encoded Rv1258c gene consistently reduces the levels of tetracycline accumulation by

B

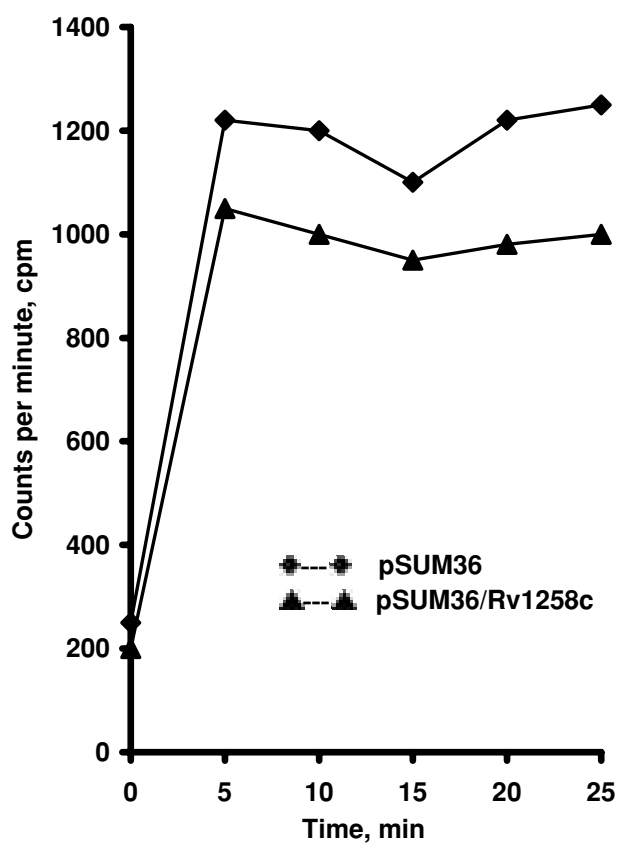

Fig. 4. (A) Accumulation of norfloxacin by M. smegmatis cells transformed with pMD31 and pMD31(Rv1634), respectively. (B) Accumulation of titriated tetracycline by M. smegmatis cells transformed with pSUM36 and pSUM36(Rv1258c). 
$10-15 \%$ (Fig. 4B), in comparison to the levels of tetracycline accumulation of cells containing only the cloning vector. This result supports the implication of the Rv1258c protein in the efflux of this antibiotic.

\section{Conclusions}

The clinical impact of antibiotic efflux pumps on resistance in MTB clinical isolates remains difficult to establish because we lack large-scale and international statistics comparing their prevalence with that of the other resistance mechanisms. This raises the question of the adequacy of the routine procedures used to detect these strains, which most often may be classified as moderately resistant and erroneously assigned to a conventional mechanism of resistance in the absence of further detailed investigation.

Further, the existence of antibiotic efflux pumps and their impact on therapy must now be taken fully into account for the selection of novel antimicrobials. The design of specific, potent inhibitors appears to be an important goal for the improved control of infectious diseases in the near future.

\section{Acknowledgments}

This research was supported by MURST-COFIN1998 and by the European Union research project "Quality of Life and Management of Living Resources" (Contract $\mathrm{N}^{\circ}$ QLK2-CT-2000-01761) and by Fondo d'Ateneó per la Ricerca 2002 (F.A.R.). J.A.A. is a recipient of a "Programa Ramón y Cajal" research fellowship (Spanish Ministry of Science and Technology). P.E.A.S. was supported by CAPES (Brazilian Ministry of Education).

\section{References}

1. Draper P. (1998) The outer parts of the mycobacterial envelope as permeability barriers. Front. Biosci. 15: 1253-1261.

2. Nikaido H. (2001) Preventing drug access to targets: cell surface permeability barriers and active efflux in bacteria. Cell Dev. Biol. 12: 215-223.

3. Kohler T, Pechere JC, Plesiat P. (1999) Bacterial efflux systems of medical importance. Cell Mol. Life Sci. 56: 771-778.

4. Saier Jr MH. (2000) A functional-phylogenetic classification system for transmembrane solute transporters. Microbiol. Mol. Biol. Rev. 64: 354-411.

5. Takiff HT, Cimino M, Musso MC, et al. (1996) Efflux pump of the proton antiporter family confers low-level fluoroquinolone resistance in Mycobacterium smegmatis. Proc. Natl. Acad. Sci. U.S.A. 93: 362-366.

6. De Rossi E, Blokpoel MC, Cantoni R, et al. (1998) Molecular cloning and functional analysis of a novel tetracycline resistance determinant, tet( $V)$, from Mycobacterium smegmatis. Antimicrob. Agents Chemother. 42: 1931-1937.

7. Aínsa JA, Blokpoel MC, Otal I, et al. (1998) Molecular cloning and characterization of Tap, a putative multidrug efflux pump present in Mycobacterium fortuitum and Mycobacterium tuberculosis. J. Bacteriol. 180: 5836-5843.

8. Silva PE, Bigi F, de La Paz Santangelo M, et al. (2001) Characterization of P55, a multidrug efflux pump in Mycobacterium bovis and Mycobacterium tuberculosis. Antimicrob. Agents Chemother. 45: 800-804.
9. Doran JL, Pang Y, Mdluli KE, et al. (1997) Mycobacterium tuberculosis efpA encodes an efflux protein of the QacA transporter family. Clin. Diagn. Lab. Immunol. 4: 23-32.

10. De Rossi E, Branzoni M, Cantoni R, et al. (1998) $m m r$, a Mycobacterium tuberculosis gene conferring resistance to small cationic dyes and inhibitors. J. Bacteriol. 180: 6068-6071.

11. Banerjee SK, Bhatt K, Misra P, et al. (2000) Involvement of a natural transport system in the process of efflux-mediated drug resistance in Mycobacterium smegmatis. Mol. Gen. Genet. 262: 949-956.

12. Braibant M, Gilot P, Content J. (2000) The ATP binding cassette (ABC) transport systems of Mycobacterium tuberculosis. FEMS Microbiol. Rev. 24: 449-467.

13. Pao SS, Paulsen IT, Saier Jr MH. (1998) Major Facilitator Superfamily. Microbiol. Mol. Biol. Rev. 62: 1-34.

14. Saier Jr MH, Paulsen IT. (2001) Phylogeny of multidrug transporters. Semin. Cell Dev. Biol. 12: 205-2 13.

15. Saier Jr MH, Beatty JT, Goffeau A, et al. (1999) The major facilitator superfamily. J. Mol. Microbiol. Biotechnol. 1: 257-279.

16. Paulsen IT, Brown MH, Skurray RA. (1996) Proton-dependent multidrug efflux systems. Microbiol. Rev. 60: 575-608.

17. Varela MF, Sansom CE, Griffith JK. (1995) Mutational analysis and molecular modelling of an amino acid sequence motif conserved in antiporters but not symporters in a transporter superfamily. Mol. Membr. Biol. 12: 313-319.

18. Cole ST, Brosch R, Parkhill J, et al. (1998) Deciphering the biology of Mycobacterium tuberculosis from the complete genome sequence. Nature 393: 537-544.

19. Bailey TL, Gribskov M. (1998) Combining evidence using p-values: application to sequence homology searches. Bioinformatics 14: 48-54.

20. Thompson JD, Higgins DG, Gibson TJ. (1994) CLUSTAL W: improving the sensitivity of progressive multiple sequence alignment through sequence weighting, position-specific gap penalties and weight matrix choice. Nucleic Acids Res. 22: 4673-4680.

21. Page RDM. (1996) TreeView: an application to display phylogenetic trees on personal computers. Comput. Appl. Biosci. 12: 357-358.

22. Altschul M, Madden TL, Schaffer AA, et al. (1997) Gapped BLAST and PSI-BLAST: a new generation of protein database search programs. Nucleic Acids Res. 25: 3389-3402.

23. Sonnhammer ELL, von Heijne G, Krogh A. (1998) A hidden Markov model for predicting transmembrane helices in protein sequences. In Glasgow J, Littlejohn T, Major F, Lathrop R, Sankoff D, Sensen C, eds. Proceedings of the Sixth International Conference on Intelligent Systems for Molecular Biology. Menlo Park, CA: AAAI Press; pp. 175-182.

24. Donnelly-Wu MK, Jacobs Jr R, Hatfull GF. (1993) Superinfection immunity of mycobacteriophage L5: applications for genetic transformation of mycobacteria. Mol. Microbiol. 7: 407-417.

25. Aínsa JA, Martín C, Cabeza M, et al. (1996) Costruction of a family of Mycobacterium/Escherichia coli shuttle vectors derived from pAL5000 and pACYC184: their use for cloning an antibiotic-resistance gene from Mycobacterium fortuitum. Gene 176: $23-26$.

26. Mortimer PGS, Piddock LJV. (1991) A comparison of methods used for measuring the accumulation of quinolones by Enterobacteriaceae, Pseudomonas aeruginosa and Staphylococcus aureus. J. Antimicrob. Chemother. 28: 639-653.

27. Williams KJ, Chung GA, Piddock LJ. (1998) Accumulation of norfloxacin by Mycobacterium aurum and Mycobacterium smegmatis. Antimicrob. Agents Chemother. 42: 795-800.

28. Sander P, De Rossi E, Boddinghaus B, et al. (2000) Contribution of the multidrug efflux pump LfrA to innate mycobacterial drug resistance. FEMS Microbiol. Lett. 193: 19-23.

29. Bush K, Goldschmidt R. (2000) Effectiveness of fluoroquinolones against gram-positive bacteria. Curr. Opin. Investig. Drugs 1: 22-30. 